\title{
GROUP ALGEBRA MODULES. III
}

\author{
BY \\ S. L. GULICK( ${ }^{(1)}$, T.-S. LIU( $\left.{ }^{1}\right)$ AND A. C. M. VAN ROOIJ
}

\begin{abstract}
Let $\Gamma$ be a locally compact group and $K$ a Banach space. The left $L^{1}(\Gamma)$ module $K$ is by definition absolutely continuous under the composition $*$ if for $k \in K$ there exist $f \in L^{1}(\Gamma), k^{\prime} \in K$ with $k=f * k^{\prime}$. If the locally compact Hausdorff space $X$ is a transformation group over $\Gamma$ and has a measure quasi-invariant with respect to $\Gamma$, then $L^{1}(X)$ is an absolutely continuous $L^{1}(\Gamma)$ module - the main object we study. If $Y \subseteq X$ is measurable, let $L_{Y}$ consist of all functions in $L^{1}(X)$ vanishing outside $Y$. For $\Omega \subseteq \Gamma$ not locally null and $B$ a closed linear subspace of $K$, we observe the connection between the closed linear span (denoted $L_{\Omega} * B$ ) of the elements $f * k$, with $f \in L_{\Omega}$ and $k \in B$, and the collection of functions of $B$ shifted by elements in $\Omega$. As a result, a closed linear subspace of $L^{1}(X)$ is an $L_{z}$ for some measurable $Z \subseteq X$ if and only if it is closed under pointwise multiplication by elements of $L^{\infty}(X)$. This allows the theorem stating that if $\Omega \subseteq \Gamma$ and $Y \subseteq X$ are both measurable, then there is a measurable subset $Z$ of $X$ such that $L_{\Omega} * L_{Y}=L_{Z}$. Under certain restrictions on $\Gamma$, we show that this $Z$ is essentially open in the (usually stronger) orbit topology on $X$. Finally we prove that if $\Omega$ and $Y$ are both relatively sigma-compact, and if also $L_{\Omega} * L_{Y} \subseteq L_{Y}$, then there exist $\Omega_{1}$ and $Y_{1}$ locally almost everywhere equal to $\Omega$ and $Y$ respectively, such that $\Omega_{1} Y_{1} \subseteq Y_{1}$; in addition we characterize those $\Omega$ and $Y$ for which $L_{\Omega} * L_{\Omega}=L_{\Omega}$ and $L_{\Omega} * L_{Y}=L_{Y}$.
\end{abstract}

1. Introduction. This paper, and the one which follows, arise quite naturally from our earlier papers [3] and [4]. Let us see how. Take $\Gamma$ as a locally compact group, and $L^{1}(\Gamma)$ the Banach space of integrable functions on $\Gamma$. If we let $K$ be an arbitrary left $L^{1}(\Gamma)$ module, we may inquire what are the left module homomorphisms from $L^{1}(\Gamma)$ to $K$. In [3], amongst other things, we give a (not quite complete) solution to the general question, and then give complete solutions in case $K=L^{p}(\Gamma)$, $p \in[1, \infty]$. In [4] we assume that $\Gamma$ acts on a given locally compact space $X$ as a transformation group and that $m_{X}$ is a measure on $X$ quasi-invariant with respect to $\Gamma$. Then we show that $L^{p}(X)$ may be rendered as a left $L^{1}(\Gamma)$ module, to which we may ask what are the left module homomorphisms from $L^{1}(\Gamma)$ to $L^{p}(X)$.

The present investigations start at that point. In this paper we discuss the more general aspects of Banach spaces $K$ which can be represented as left $L^{1}(\Gamma)$ modules. We denote the module composition by $*$. We pay particular attention to those modules whose elements are factorable (i.e., $k \in K$ implies that there is an

Received by the editors June 17, 1969.

AMS 1969 subject classifications. Primary 4680, 2220.

Key words and phrases. Transformation group, quasi-invariant measure, absolutely continuous measure, approximate identity, factorable, orbit topology, vanishing algebra, group algebra module.

(1) Research supported in part by the National Science Foundation. 
$f \in L^{1}(\Gamma)$ and $k^{\prime} \in K$ such that $\left.k=f * k^{\prime}\right)$. Such spaces we call absolutely continuous modules. For each element in such a $K$ we can describe the notion of left shift by elements of $\Gamma$, and each such shift by $\sigma \in \Gamma$ is continuous as a function of $\sigma$.

A major reason for our study of absolutely continuous modules appears in $\$ 3$. For $\Omega \subseteq \Gamma$, let $L_{\Omega}$ consist of all $L^{1}(\Gamma)$ functions vanishing off $\Omega$. If $B$ is a closed subspace of $K$, we denote by $L_{\Omega} * B$ the closed linear span of elements of the form $f * k, f \in L_{\Omega}$ and $k \in K$. The fundamental Decomposition Theorem 3.2 states that if $\Omega$ is not locally null, then any such $L_{\Omega} * B$ can be approximated by sums of shifts of $B$ by elements essentially (to be made precise in the text) in $\Omega$. If $Y$ is measurable in $X$, and $L_{Y}$ has a meaning analogous to $L_{\Omega}$, then $L_{\Omega} * L_{Y}$ may be approximated by sums of shifts of $L_{Y}$. In particular, $L_{\Gamma} * L_{\Omega}=L_{\Gamma}$, the whole space! Together with Theorem 3.9, which determines that a closed linear subspace of $L^{1}(X)$ is an $L_{Z}$ for some measurable $Z \subseteq X$ if and only if it is closed under pointwise multiplication by $L^{\infty}(X)$, these results allow us to prove in Theorem 3.10 that if $\Omega \subseteq \Gamma$ and $Y \subseteq X$ are both measurable, then there exists a measurable subset $Z$ of $X$ such that $L_{\Omega} * L_{Y}=L_{Z}$. We terminate the section with a sort of counterpart to the decomposition and a corollary of value in later studies.

In the rest of this paper we analyze the set $Z$ occurring above-under the stipulation that if $\Gamma_{0}$ is a sigma-compact open subgroup of $\Gamma$, then $m_{X}\left(\Gamma_{0} x\right)>0$ for all $x \in X$. This condition gives us certain continuity conditions on the convolution which we utilize, and without the condition $Z$ is unmanageable. It turns out that $Z$ is essentially open-in a certain natural topology which ordinarily is stronger than the given topology on $X$. This new topology we call the orbit topology since it is described in terms of the orbits $\Gamma x, x \in X$, and we discuss it in $\$ 4$ when we are in the process of determining $Z$.

In $\$ 5$ we study the relationship between the two notions $\Omega Y \subseteq Y$ and $L_{\Omega} * L_{Y} \subseteq L_{Y}$. That $\Omega Y \subseteq Y$ implies $L_{\Omega} * L_{Y} \subseteq L_{Y}$ is true and easy to prove. The converse would say that if $L_{\Omega} * L_{Y} \subseteq L_{Y}$ then there exist $\Omega_{1}, Y_{1}$ l.a.e. equal to $\Omega, Y$ respectively, such that $\Omega_{1} Y_{1} \subseteq Y_{1}$. It is as yet not known, even if $X=\Gamma$ and $Y=\Omega$, except when $\Omega$ is relatively sigma-compact [6]. Nevertheless, we prove it when both $\Omega$ and $Y$ are relatively sigma-compact. We conclude the paper by characterizing those $\Omega$ and $Y$ for which $L_{\Omega} * L_{\Omega}=L_{\Omega}$ and $L_{\Omega} * L_{Y}=L_{Y}$.

2. Setting. We begin the definitions and notations by prescribing $\varnothing$ to be the empty set. If $A$ and $B$ are two sets, then $B \backslash A$ is the complement of $A \cap B$ in $B$. Let $\Gamma$ be a locally compact group with identity $1, m$ a left Haar measure on $\Gamma$, and $L_{\Gamma}$ the Banach space of integrable functions on $\Gamma$, with the usual norm \|\|$_{1}$. For $\sigma \in \Gamma$ and $f \in L_{\Gamma}$ we have the left shift $f_{\sigma}$ (also in $L_{\Gamma}$ ), defined by $f_{\sigma}(\tau)=f(\sigma \tau), \tau \in \Gamma$, and the right shift $f^{\sigma}$ (in $L_{\Gamma}$ ), defined by $f^{\sigma}(\tau)=f(\tau \sigma) \Delta(\sigma), \tau \in \Gamma$, where $\Delta$ is the modular function for $\Gamma$. For $f \in L_{\Gamma}$ we let $f^{\prime} \in L_{\Gamma}$ be defined by $f^{\prime}(\tau)=\Delta\left(\tau^{-1}\right) f\left(\tau^{-1}\right)$. If $\Omega \subseteq \Gamma$, we let $L_{\Omega}$ denote the collection of functions in $L_{\Gamma}$ which vanish almost everywhere in $\Gamma \mid \Omega$. 
Let $X$ be a locally compact (Hausdorff) space. We say that $\Gamma$ acts as a transformation group on $X$ if $\Gamma$ is a group of homeomorphisms on $X$ such that the map $\Gamma \times X \rightarrow X$ defined by $(\sigma, x) \rightarrow \sigma x, \sigma \in \Gamma, x \in X$, is jointly continuous. If $m_{X}$ is a positive Radon measure on $X$ with the property that if $Y \subseteq X$ and $m_{X}(Y)=0$, then $m_{X}(\sigma Y)=0$ for all $\sigma \in \Gamma$, then we say that $m_{X}$ is quasi-invariant. The functions integrable on $X$ with respect to $m_{X}$ describe the space $L_{X}$, under the usual $L^{1}$ norm. For $Y \subseteq X$ and $p \in[1, \infty], L_{Y}^{p}=\left\{f \in L^{p}(X): f=0\right.$ l.a.e. on $\left.X \backslash Y\right\}$. The characteristic function of $Y \subseteq X$ is written $\xi_{Y}$. We abbreviate "almost everywhere" to "a.e.", and "locally almost everywhere" to "l.a.e." We use the notation $Y \subseteq Z$ 1.a.e. to mean that $Z \backslash Y$ is locally null. Then $Y=Z$ l.a.e. means that $Y \subseteq Z$ l.a.e. and $Z \subseteq Y$ 1.a.e. For measure-theoretic notations we generally follow [5]. Let $L_{X}^{\infty}$ denote the measurable, essentially bounded functions on $X$. We let $I$ denote an indexing set (to serve the purpose). We denote by $\boldsymbol{R}$ the additive group of real numbers with the usual topology.

Throughout the paper $K$ will denote a Banach space, $K^{*}$ the topological conjugate (dual) space under its usual dual norm. For every $\mu \in M(\Gamma)$ and every bounded continuous map $F: \Gamma \rightarrow K$ there exists by Proposition 8 of $\S 1$ of [1] a unique element $\int F d \mu \in K$ such that

$$
k^{*}\left(\int F d \mu\right)=\int_{x}\left(k^{*} \circ F\right) d \mu, \quad k^{*} \in K^{*}
$$

Further, if $K_{1}$ is another Banach space and $T: K \rightarrow K_{1}$ is a continuous linear map, then $\int(T \circ F) d \mu=T\left(\int F d \mu\right)$.

If $f \in L_{\Gamma}$, there is a unique $\mu \in M(\Gamma)$ with $d \mu(\sigma)=f(\sigma) d \sigma$, $d \sigma$ representing the element of the left invariant Haar measure. Instead of $\int F d \mu$ we shall write $\int f(\sigma) F(\sigma) d \sigma$. Then $\left\|\int f(\sigma) F(\sigma) d \sigma\right\| \leqq \int_{\Gamma}|f(\sigma)| \cdot\|F(\sigma)\| d \sigma$.

Let $K$ be a Banach space. We call $(K, *)$ a left $L_{\Gamma}$-module if $K$ is a Banach space over the same scalar field as $L_{\Gamma}$ and if $*$ is a bilinear operation with the following properties:

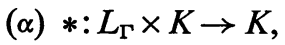

(B) $(f * g) * k=f *(g * k), f, g \in L_{\Gamma}, k \in K$,

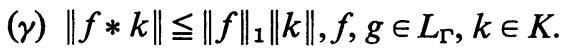

We should not confuse the module composition with convolution, even though they are given by the same symbol. The former acts on $L_{\Gamma} \times K$, and the latter on $L_{\Gamma} \times L_{\Gamma}$. A simple glance to the right and left of $*$ should tell which space $*$ acts on.

For any $L_{\Gamma}$-module $K$ let us denote by $K_{\text {abs }}$ the space $\left\{f * k: f \in L_{\Gamma}, k \in K\right\}$. Then $K_{\text {abs }}$ is a closed submodule of $K$ (Corollary 2.3 of [4]), and $\left(K_{\mathrm{abs}}\right)_{\mathrm{abs}}=K_{\mathrm{abs}}$. (This follows from the fact that $L_{\Gamma}$ is factorable; see [2].) If $K=K_{\text {abs }}$, we say that $K$ is absolutely continuous. It is easy to see that if $K$ is absolutely continuous, then for an approximate identity $\left(u_{i}\right)_{i \in I}$ in $L_{\mathrm{I}}$, we have $\lim _{i}\left(u_{i} * k\right)=k$ for each $k \in K$. As $K_{\text {abs }}$ is closed, the converse is also true. 
If $K$ is absolutely continuous, then every element $\sigma \in \Gamma$ defines a unique linear isometry $k \rightarrow k_{\sigma}$ of $K$ onto $K$ such that

$$
\begin{aligned}
f * k & =\int f(\tau) k_{\tau-1} d \tau, \\
(f * k)_{\sigma} & =f_{\sigma} * k, \text { and } f * k_{\sigma}=f^{\sigma} * k \quad\left(f \in L_{\Gamma}, k \in K, \sigma \in \Gamma\right) .
\end{aligned}
$$

$k_{\sigma}$ is called the shift of $k$ by $\sigma$. It is jointly continuous and satisfies $\left(k_{\sigma}\right)_{\tau}=k_{\sigma \tau}$, $k_{1}=k$. We shall omit the proof of these facts. We only mention that $k_{\sigma}$ may be defined by $k_{\sigma}=\lim _{i} u_{i}^{\sigma} * k(k \in K)$.

We defend the terminology "absolutely continuous module" by noting that if $K=M(\Gamma)$, then $\mu \in M(\Gamma)$ is absolutely continuous in the conventional sense (with respect to left Haar measure) if and only if $\mu \in M(\Gamma)_{\text {abs }}$.

We describe other examples of $L_{\Gamma}$-modules, some of which we will find are absolutely continuous, and others not. To begin with, let $\Gamma$ be a locally compact transformation group acting on a locally compact Hausdorff space $X$, as described above. We make $C_{\infty}(X)$ and $M(X)$ into $L_{\Gamma}$-modules by

$$
\begin{aligned}
& f * k(x)=\int_{\Gamma} f(\sigma) k\left(\sigma^{-1} x\right) d \sigma \quad\left(f \in L_{\Gamma}, k \in C_{\infty}(X), x \in X\right) \\
& f * \mu(k)=\int_{\Gamma}\left(f^{\prime} * k\right) d \mu \quad\left(f \in L_{\Gamma}, \mu \in M(X), k \in C_{\infty}(X)\right) .
\end{aligned}
$$

$C_{\infty}(X)$ is absolutely continuous $([4,4.11])$ and the shift is given by

$$
k_{\sigma}(x)=k(\sigma x) \quad\left(\sigma \in \Gamma, x \in X, k \in C_{\infty}(X)\right) .
$$

In general, $M(X)$ will not be absolutely continuous. The space $M(X)_{\mathrm{abs}}$ has been discussed in [7].

Let $\Gamma$ act on $X$ as above, and let $m_{X}$ be a quasi-invariant measure on $X$. The realization of $L_{X}$ as the space of all elements of $M(X)$ that are absolutely continuous with respect to $m_{X}$ makes $L_{X}$ an absolutely continuous submodule of $M(X)$ (Theorem 4.11 of [4]). In $[4, \S 4]$ the authors have constructed a positive measurable function $J$ on $\Gamma \times X$ such that for $f \in L_{\Gamma}$ and $k \in L_{X}$,

$$
f * k(x)=\int_{\Gamma} f(\sigma) k\left(\sigma^{-1} x\right) J\left(\sigma^{-1}, x\right) d \sigma
$$

for locally almost all $x \in X$. By means of this $J$ we can make every $L_{X}^{p}(1 \leqq p \leqq \infty)$ into an $L_{\Gamma}$-module by defining

$$
\left.f * k(x)=\int_{\Gamma} f(\sigma) k\left(\sigma^{-1} x\right) J\left(\sigma^{-1}, x\right)^{p^{-1}} d \sigma \quad \text { (1.a.e. } x \in X\right)
$$

for all $f \in L_{\Gamma}, k \in L_{X}^{p}$. As was shown in [4, Theorem 4.11] $L_{X}^{p}$ is absolutely continuous if $p<\infty$. Generally $L_{X}^{\infty}$ is not. The canonical map $C_{\infty}(X) \rightarrow L_{X}^{\infty}$ is a module homomorphism. In particular, if supp $m_{X}=X, C_{\infty}(X)$ is an absolutely continuous submodule of $L_{X}^{\infty}$. 
One final note on concrete examples of $L_{\Gamma}$ absolutely continuous modules. There exist examples for which the composition is not a generalized convolution. Take $X$ to be an abelian locally compact group and let $\Gamma$ be the character group of $X$. For $f \in L_{\Gamma}$ and $k \in L_{X}$, put $f * k=\hat{f} k$.

Absolutely continuous $L_{\Gamma}$-modules have some inheritance properties. In the first place, we have already mentioned that closed submodules of absolutely continuous $L_{\Gamma}$-modules are themselves absolutely continuous. Next we come to sums and intersections of absolutely continuous $L_{\Gamma}$-modules, which we define forthwith. Let $K_{1}$ and $K_{2}$ be $L_{\Gamma}$-modules and let $H$ be a closed subspace of the product $K_{1} \times K_{2}$. We require that $\left(f * k_{1}, f * k_{2}\right) \in H$ for any $f \in L_{\Gamma},\left(k_{1}, k_{2}\right) \in H$. The vector spaces $H$ and $\left(K_{1} \times K_{2}\right) / H$ are turned into Banach spaces $K_{1} \wedge_{H} K_{2}$ and $K_{1} \vee_{H} K_{2}$, respectively, by the definitions

$$
\begin{aligned}
\left\|\left(k_{1}, k_{2}\right)\right\| & =\max \left(\left\|k_{1}\right\|,\left\|k_{2}\right\|\right), \\
\left\|\left(k_{1}, k_{2}\right)+H\right\| & =\inf \left\{\left\|k_{1}^{\prime}\right\|+\left\|k_{2}^{\prime}\right\|: k_{1}^{\prime} \in K_{1}, k_{2}^{\prime} \in K_{2} \text { and }\left(k_{1}^{\prime}, k_{2}^{\prime}\right) \equiv\left(k_{1}, k_{2}\right)+H\right\} .
\end{aligned}
$$

( $K_{1} \wedge_{H} K_{2}$ is called the intersection of $K_{1}$ and $K_{2} ; K_{1} \vee_{H} K_{2}$ is their sum.)

The proofs of these facts and a general investigation of these Banach spaces occur in [8]. Carrying on, we render $K_{1} \wedge_{H} K_{2}$ and $K_{1} \vee_{H} K_{2}$ as $L_{\Gamma}$-modules by the quite natural formulas

$$
\begin{aligned}
f *\left(k_{1}, k_{2}\right) & =\left(f * k_{1}, f * k_{2}\right), & & f \in L_{\Gamma},\left(k_{1}, k_{2}\right) \in H, \\
f *\left[\left(k_{1}, k_{2}\right)+H\right] & =\left(f * k_{1}, f * k_{2}\right)+H, & & f \in L_{\Gamma}, k_{1} \in K_{1}, k_{2} \in K_{2} .
\end{aligned}
$$

Furthermore, if $K_{1}$ and $K_{2}$ are absolutely continuous, then so are $K_{1} \wedge_{H} K_{2}$ and $K_{1} \vee{ }_{H} K_{2}$. In fact, it is simple to compute that

$$
\begin{aligned}
& \left(K_{1} \wedge_{H} K_{2}\right)_{\mathrm{abs}}=H \cap\left\{\left(k_{1}, k_{2}\right): k_{1} \in\left(K_{1}\right)_{\mathrm{abs}}, k_{2} \in\left(K_{2}\right)_{\mathrm{abs}}\right\}, \\
& \left(K_{1} \vee_{H} K_{2}\right)_{\mathrm{abs}}=\left\{\left(k_{1}, k_{2}\right)+H: k_{1} \in\left(K_{1}\right)_{\mathrm{abs}}, k_{2} \in\left(K_{2}\right)_{\mathrm{abs}}\right\} .
\end{aligned}
$$

If we wish to investigate the dual $K^{*}$ of an $L_{\Gamma}$-module $K$, a natural composition is defined by

$$
\left(f * k^{*}\right) k=k^{*}\left(f^{\prime} * k\right), \quad f \in L_{\Gamma}, k \in K, k^{*} \in K^{*},
$$

and endowed with it, $K^{*}$ is an $L_{\Gamma}$-module. We have already used this formula to define a module structure on $M(X)=C_{\infty}(X)^{*}$. In general, $K^{*}$ will not be absolutely continuous, even if $K$ is. For example, take $K=L_{X}$ where $X=\Gamma=R$. Then $K$ is absolutely continuous, while $K^{*}=L^{\infty}(X)$ is not. On the other hand, if we use the criterion for absolute continuity of $K$ that $K$ must be factorable, then an application of the Hahn-Banach theorem shows us that if $K$ is not absolutely continuous, then under no circumstance can $K^{*}$ be. In fact $K$ is absolutely continuous if and only if $K^{*}$ is order-free. (An $L_{\Gamma}$-module $K$ is said to be order-free if for each $k \in K, f * k=0$ for all $f \in L_{\Gamma}$ implies $k=0$.) For a corollary we observe that all reflexive order-free modules are absolutely continuous and have absolutely continuous duals. 
3. A decomposition theorem and its consequences. Before we can give the decomposition theorem in the form we desire, we must have a preliminary discussion. Assume that $X$ is locally compact and Hausdorff and has a positive Radon measure $m_{X}$. For a measurable set $Y$ we define the two operators $i$ and $d$ as follows:

$i Y=\{x \in X:$ there exists a measurable neighborhood

$$
\left.V \text { of } x \text { such that } m_{X}(V \backslash Y)=0\right\},
$$

$d Y=\left\{x \in X:\right.$ for every measurable neighborhood $V$ of $\left.x, m_{X}(V \cap Y)>0\right\}$.

The operators $i$ and $d$ are not new; they have been discussed in [6]. Their more elementary properties are:

$Y^{0} \subseteq i Y=(i Y)^{0} \subseteq d Y=(\mathrm{Cl}(d Y)) \subseteq \bar{Y}, i Y \subseteq Y$ 1.a.e., and $Y \subseteq d Y$ l.a.e.

$\sigma(i Y)=i(\sigma Y)$ and $\sigma(d Y)=d(\sigma Y)$ for every $\sigma \in \Gamma$.

$Y \subseteq Y^{\prime}$ l.a.e. implies $i Y \subseteq I Y^{\prime}$ and $d Y \subseteq d Y^{\prime}$.

Verbally, $i Y$ is an open set containing the interior of $Y$, while $d Y$ is a closed set contained in the closure of $Y$.

In particular, the operators $d$ and $i$ are defined in $\Gamma$ itself. One of the basic properties of $d$ is the following.

3.1. Lemma. Let $\Omega \subseteq \Gamma$ be measurable, $\gamma \in d \Omega$. Then $L_{\Gamma}$ contains an approximate identity $\left(u_{i}\right)_{i \in I}$ such that $\left\|u_{i}\right\|_{1}=1$ and $\left(u_{i}\right)^{\gamma^{-1}} \in L_{\Omega}$ for every $i$.

Proof. For $I$ we take the net of all compact neighborhoods of $1 \in \Gamma$, made into a directed set by the definition $\Phi_{1} \prec \Phi_{2}$, if $\Phi_{1} \supseteq \Phi_{2}$. For $\Phi \in I$ let

$$
u_{\Phi}=\left[m\left(\Phi \cap \Omega \gamma^{-1}\right)\right]^{-1} \xi_{\Phi \cap \Omega \gamma^{-1}} \text {. }
$$

(Note that $m\left(\Phi \cap \Omega \gamma^{-1}\right) \neq 0$ because $\gamma \in d \Omega$.) Then $\left(u_{\Phi}\right)^{\gamma-1} \in L_{\Omega}$ and $\left\|u_{\Phi}\right\|_{1}=1$. Take $f \in L_{\Gamma}, \varepsilon>0$. The set $\Phi_{1}=\left\{\sigma \in \Gamma:\left\|f_{\sigma}-1-f\right\|<\varepsilon\right\}$ is a neighborhood of $1 \in \Gamma$. It is now easy to see that $\left\|\left(u_{\Phi} * f\right)-f\right\|_{1}<\varepsilon$ for all $\Phi \in I$ such that $\Phi \subseteq \Phi_{1}$. In fact, for such $\Phi$,

$$
\begin{aligned}
\left\|\left(u_{\Phi} * f\right)-f\right\|_{1} & =\left\|\int_{\Gamma} u_{\Phi}(\sigma) f_{\sigma^{-1}} d \sigma-\int_{\Gamma} u_{\Phi}(\sigma) f d \sigma\right\| \\
& \leqq \int_{\Gamma} u_{\Phi}(\sigma)\left\|f_{\sigma}-1-f\right\| d \sigma<\varepsilon .
\end{aligned}
$$

Let $B$ be a closed linear subspace of an absolutely continuous $L_{\Gamma}$-module $K$. For $\sigma \in \Gamma$ we denote $\left\{k_{\sigma}: k \in B\right\}$ by $B_{\sigma}$. For $\Omega \subseteq \Gamma$ measurable we indicate by $L_{\Omega} * B$ the closed linear subspace of $K$ generated by $\left\{f * k: f \in L_{\Omega}, k \in B\right\}$. If $\Gamma=X$ and $B \subseteq L_{X}$ let $B^{\prime}=\left\{f^{\prime} \in L_{X}: f \in B\right\}$. In particular, if $Y \subseteq X=\Gamma$, then $L_{Y^{-1}} * L_{\Omega^{-1}}=\left(L_{Y}\right)^{\prime} *\left(L_{\Omega}\right)^{\prime}$ $=\left(L_{\Omega} * L_{Y}\right)^{\prime}$ by direct computation.

Now we are ready for the decomposition theorem.

3.2. MOdULE. DECOMPOSITION THEOREM. Let $K$ be an absolutely continuous module over $L_{\Gamma}$. For any measurable $\Omega \subseteq \Gamma$ and any closed subspace $B$ of $K$ we have

$$
L_{\Omega} * B=\mathrm{Cl}\left(\sum_{\sigma \in d \Omega} B_{\sigma}^{-1}\right)
$$


Proof. Let $\sigma \in d \Omega$ and $k \in B$. By Lemma 3.1 there is an approximate identity $\left(u_{i}\right)_{i \in I}$ in $L_{\Gamma}$ such that $\left(u_{i}\right)^{\sigma^{-1}} \in L_{\Omega}$ for every $i$. Then $k_{\sigma^{-1}}=\lim _{i}\left(\left(u_{i}\right)^{\sigma^{-1}} * k\right) \in L_{\Omega} * B$. Thus $\mathrm{Cl}\left(\sum_{\sigma \in d \Omega} B_{\sigma^{-1}}\right) \subseteq L_{\Omega} * B$. Conversely, let $f \in L_{\Omega}, k \in B$. For locally almost all

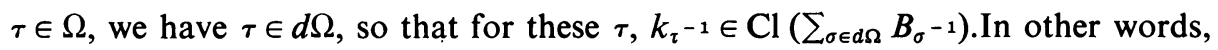
$k \in B$ implies $k_{\tau^{-1}} \in \mathrm{Cl}\left(\sum_{\sigma \in d \Omega} B_{\sigma^{-1}}\right)$ for locally almost every $\tau \in \Omega$. Take any $k^{*} \in K^{*}$ such that $k^{*}=0$ on $\sum_{\sigma \in d \Omega} B_{\sigma^{-1}}$. Then $k^{*}(f * k)=\int_{\Gamma} f(\sigma) k^{*}\left(k_{\sigma}-1\right) d \sigma=0$ for all $f \in L_{\Omega}, k \in B$, with the result that $k^{*}=0$ on $L_{\Omega} * B$. Thus $L_{\Omega} * B \subseteq \mathrm{Cl}\left(\sum_{\sigma \in d \Omega} B_{\sigma^{-1}}\right)$.

We mention that without the hypothesis of absolute continuity on $K$ the conclusion may be invalid. Take, for example, $\Gamma=X=R$. Let $B=K=L_{X}^{\infty}$. Then for each $\sigma \in \Gamma, B_{\sigma^{-1}}=L_{X}^{\infty}$, while $L_{\Omega} * B$ is the collection of uniformly continuous functions on $X$.

Several consequences follow directly.

\subsection{Corollary. $L_{\Omega} * B=L_{d \Omega} * B$.}

Corollaries 3.4 and 3.5 concern the case where $\Gamma$ is a transformation group acting on $X$ and $X$ is endowed with a quasi-invariant measure. For $Y \subseteq X$ measurable and $\sigma \in \Gamma$ we have $\left(L_{Y}\right)_{\sigma^{-1}}=L_{\sigma Y}$. Thus

3.4. Corollary. If $Y \subseteq X$ is measurable, then $L_{\Omega} * L_{Y}=\mathrm{Cl}\left(\sum_{\sigma \in d \Omega} L_{\sigma Y}\right)$.

3.5. Corollary. Let $Y$ and $Z$ be measurable subsets of $X$ and let $L_{\Omega} * L_{Y} \subseteq L_{Z}$. Then

(i) For every $\sigma \in d \Omega, \sigma Y \subseteq Z$ l.a.e.

(ii) $d \Omega d Y \subseteq d Z$, so that if $L_{\Omega}$ is a subalgebra of $L_{\Gamma}$, then $d \Omega$ is a subsemigroup of $\Gamma$.

(iii) $d \Omega i Y \subseteq i Z$, so that if $L_{\Omega}$ is a subalgebra of $L_{\Gamma}$, then $i \Omega$ is a subsemigroup of $\Gamma$.

If , in addition, $X=\Gamma$, then also

(iv) For every $\sigma \in d Y, \Omega \sigma \subseteq Z$ l.a.e.

(v) $i \Omega d Y \subseteq i Z$.

Proof. By the preceding corollary, if $\sigma \in d \Omega$, then $L_{\sigma Y} \subseteq L_{Z}$, so that $\sigma Y \subseteq Z$ l.a.e., proving (i). Then $\sigma(d Y)=d(\sigma Y) \subseteq d Z$, and $\sigma(i Y)=i(\sigma Y) \subseteq i Z$, thus proving both (ii) and (iii). Parts (iv) and (v) follow from (i) and (ii) via the formulas

$$
L_{Y^{-1}} * L_{\Omega^{-1}}=\left(L_{Y}\right)^{\prime} *\left(L_{\Omega}\right)^{\prime}=\left(L_{\Omega} * L_{Y}\right)^{\prime} \subseteq\left(L_{Z}\right)^{\prime}=L_{Z}{ }^{-1} .
$$

For later use we file away yet another consequence.

3.6. CoROllaRy. If $\Omega \subseteq \Gamma$ is measurable and not locally null, then $L_{\Gamma} * L_{\Omega}=L_{\Gamma}$.

Proof. Since $\Omega$ is not locally null, $d \Omega \neq \varnothing$, so let $\tau \in d \Omega$. Then

$$
\left(L_{\Gamma} * L_{\Omega}\right)^{\prime}=\left(L_{\Omega}\right)^{\prime} *\left(L_{\Gamma}\right)^{\prime}=L_{\Omega^{-1}} * L_{\Gamma}=\mathrm{Cl}\left(\sum_{\sigma \in d \Omega} L_{\sigma^{-1} \Gamma}\right) \supseteq L_{\tau}^{-1} \Gamma=L_{\Gamma} .
$$

The space $B$ employed in the last two corollaries has been contained in $L_{X}$. For a moment let us switch our attention to $C_{Y}$, the collection of all functions $k \in C_{\infty}(X)$ which vanish outside $Y$. Then we have 
3.7. Corollary. $L_{\Omega} * C_{Y}=\mathrm{Cl}\left(\sum_{\sigma \in d \Omega} C_{\sigma Y}\right)=L_{d \Omega} * C_{Y}$.

3.8. Corollary. If $Y$ is open in $X$, then $L_{\Omega} * C_{Y}=C_{(d \Omega) Y}$.

Proof. Since evidently $\mathrm{Cl}\left(\sum_{\sigma \in d \Omega} C_{\sigma Y}\right) \subseteq C_{(d \Omega) Y}$, we need only prove the opposite inclusion. To this end, let $f \in C_{(d \Omega) Y}$ with compact support. Since $Y$ is open and $\operatorname{supp} f$ is compact, there exist $\tau_{1}, \ldots, \tau_{n} \in d \Omega$ such that $\operatorname{supp} f \subseteq \bigcup_{i=1}^{n} \tau_{i} Y$. By using a partition of unity one can construct $f_{1}, \ldots, f_{n} \in C(X)$ with $\sum_{i=1}^{n} f_{i}=f$, such that each $f_{i}$ has compact support contained in $\tau_{i} Y$. Then $f \in \sum_{i=1}^{n} C_{\tau_{i} Y} \subseteq \sum_{\sigma \in d \Omega} C_{\sigma Y}$.

Because of Corollary 3.8, we quite involuntarily might conjecture that at least when $Y$ is open in $X$, then $L_{\Omega} * L_{Y}=L_{(d \Omega) Y}$. In fact this is true, but the proof is by no means trivial. First we show that $L_{\Omega} * L_{Y}$ is an $L_{Z}$ for an appropriate $Z$. For completeness we prove the following theorem.

3.9. THEOREM. Let $m_{X}$ be a positive Radon measure on a locally compact space $X$. Let $B$ be a closed linear subspace of $L_{X}$. Then the following conditions are equivalent:

(a) There is a measurable set $Z \subseteq X$ such that $B=L_{Z}$.

(b) For all $k \in B$ and $j \in L_{X}^{\infty}, k j \in B$ (i.e., $B$ is a module over $L_{\infty}(X)$ under pointwise multiplication).

Proof. The implication (a) to (b) is evident. Now assume (b). By Theorem 11.39 of [5] there exists a family $\mathscr{F}$ of disjoint compact subsets of $X$ such that for every $U$ which is open in $X$ and has finite measure, $\left\{F \in \mathscr{F}: m_{X}(U \cap F)>0\right\}$ is countable, and such that $X \bigcup \mathscr{F}$ is locally null. It follows that a set $Y \subseteq X$ is measurable if and only if $Y \cap F$ is measurable for every $F \in \mathscr{F}$. For each $F \in \mathscr{F}$ let $\mathscr{X}_{F}=\{Y \subseteq F: Y$ is measurable and $\xi_{Y} \in B$. Consequently,

$(\alpha)$ If $Y_{1}, Y_{2}, \ldots$ is a sequence in $\mathscr{X}_{F}$, then $\bigcup_{n=1}^{\infty} Y_{n} \in \mathscr{X}_{F}$.

( $\beta$ ) If a measurable set $Y$ is contained in an element of $\mathscr{X}_{F}$, then $Y \in \mathscr{X}_{F}$.

By $(\alpha)$ for every $F$ there exists a $Z_{F} \in \mathscr{X}_{F}$ such that $m_{X}\left(Z_{F}\right)=\sup \left\{m_{X}(Y): Y \in \mathscr{X}_{F}\right\}$. Then $Z=\bigcup\left\{Z_{F}: F \in \mathscr{F}\right\}$ is measurable by the comments above, and is the subset of $X$ we desire. Now we show that $B=L_{z}$. Inasmuch as both $B$ and $L_{z}$ are closed modules over $L_{X}^{\infty}$ under pointwise multiplication, it suffices to show that $\left\{Y \subseteq X: \xi_{Y} \in B\right\}=\left\{Y \subseteq X: \xi_{Y} \in L_{Z}\right\}$. To show it, first let $\xi_{Y} \in L_{Z}$ and assume that $Y \subseteq Z$ everywhere. Then for every $F \in \mathscr{F}$, we have $Y \cap F \subseteq Z \cap F=Z_{F}$, so that by $(\beta), Y \cap F \in \mathscr{X}_{F}$. Thus $\xi_{\mathrm{Y} \cap F} \in B$. Since $Y$ is of finite measure, there can exist only countably many $F \in \mathscr{F}$ such that $m_{X}(Y \cap F)>0$. Hence $\xi_{Y}=\sum\left\{\xi_{Y \cap F}: F \in \mathscr{F}\right\} \in B$. On the other hand, let $\xi_{Y} \in B$. For each $F \in \mathscr{F}, \xi_{Y \cap F}=\xi_{Y} \xi_{F} \in B$, so that $Y \cap F \in \mathscr{X}_{F}$. Then $(Y \cap F) \cup Z_{F} \in \mathscr{X}_{F}$ by $(\alpha)$. It follows that $m_{X}\left((Y \cap F) \cup Z_{F}\right) \leqq m_{X}\left(Z_{F}\right)$. Hence, $Y \cap F \subseteq Z_{F}$ a.e., which means that $Y \subseteq Z$ l.a.e., and $\xi_{Y} \in L_{Z}$.

In 3.10-3.13, $\Gamma$ is again a group of homeomorphisms of a space $X$ on which we have a quasi-invariant measure $m_{X}$.

3.10. THEOREM. For any measurable $\Omega \subseteq \Gamma$ and $Y \subseteq X$ there exists a measurable set $Z \subseteq X$ such that $L_{\Omega} * L_{Y}=L_{Z}$. 
Proof. Since each $L_{\sigma Y}$ is a module over $L_{X}^{\infty}$, for $\sigma \in d \Omega$, this means that $\mathrm{Cl}\left(\sum_{\sigma \in d \Omega} L_{\sigma Y}\right)=L_{\Omega} * L_{Y}$ is also an $L_{X}^{\infty}$ module.

We now arrive at the proposition promised following Corollary 3.8.

3.11. Corollary. If $\Omega \subseteq \Gamma$ is measurable and $Y \subseteq X$ is open, then $L_{\Omega} * L_{Y}=L_{(d \Omega) Y}$.

Proof. Let $Z$ be as in the preceding theorem. Since $Y$ is open, $Y \subset i Y$. By Corollary 3.5(iii), $d \Omega Y \subset d \Omega i Y \subset i Z$. Since always $i Z \subseteq Z$ 1.a.e., we have $(d \Omega) Y \subseteq Z$ 1.a.e. On the other hand, $L_{\Omega} * L_{Y} \subseteq L_{(d \Omega) Y}$, so that $Z \subseteq(d \Omega) Y$ 1.a.e. Consequently, $Z=(d \Omega) Y$ l.a.e., which is what we needed to prove.

With an added hypothesis we can go a step further.

3.12. TheOREM. Let $\Omega \subseteq \Gamma$ be measurable and $Y \subseteq X$ open, and assume that $L_{\Omega} * L_{Y} \subseteq L_{Y}$. Then there exist an $\Omega^{\prime} \subseteq \Gamma$ and an open $Y^{\prime} \subseteq X$ such that $\Omega^{\prime}=\Omega$ l.a.e. and $Y^{\prime}=Y$ l.a.e., and $\Omega^{\prime} Y^{\prime} \subseteq Y^{\prime}$.

Proof. Let $\Omega^{\prime}=\Omega \cap d \Omega$ and $Y^{\prime}=i Y$. Then $\Omega^{\prime}=\Omega$ l.a.e. and $Y^{\prime}=Y$ l.a.e. By the preceding corollary, $L_{(d \Omega) Y^{\prime}}=L_{\Omega} * L_{Y^{\prime}} \subseteq L_{Y^{\prime}}$, so that $\left(d \Omega^{\prime}\right) Y^{\prime} \subseteq Y^{\prime}$ 1.a.e. Note that since $Y^{\prime}$ is open, $\left(d \Omega^{\prime}\right) Y^{\prime}$ is also open. Then $\Omega^{\prime} Y^{\prime} \subseteq\left(d \Omega^{\prime}\right) Y^{\prime} \subseteq i\left\{\left(d \Omega^{\prime}\right) Y^{\prime}\right\} \subseteq i Y^{\prime}$ $=i(i Y)=i Y=Y^{\prime}$.

There is a companion to this corollary-for $Y$ closed in $X$-which we presently demonstrate.

3.13. TheOREM. Let $\Omega \subseteq \Gamma$ be measurable and $Y \subseteq X$ closed, and assume that $L_{\Omega} * L_{Y} \subseteq L_{Y}$. Then there exist a set $\Omega^{\prime} \subseteq \Gamma$ and a closed set $Y^{\prime} \subseteq X$ such that $\Omega^{\prime}=\Omega$ l.a.e. and $Y^{\prime}=Y$ l.a.e., and $\Omega^{\prime} Y^{\prime} \subseteq Y^{\prime}$.

Proof. Let $\Omega^{\prime}=\Omega \cap d \Omega$ and $Y^{\prime}=d Y$. By assumption, the $Z$ of Theorem 3.10 has the property that $Z \subseteq Y$ l.a.e. Thus $d Z \subseteq d Y$, whereupon $\Omega^{\prime} Y^{\prime} \subseteq d \Omega d Y \subseteq d Z$ $\subseteq d Y=Y^{\prime}$, by an application of Corollary 3.5(ii).

Theorem 3.10 says that if we are given measurable sets $\Omega \subseteq \Gamma$ and $Y \subseteq X$, then the collection of all $k \in L_{X}$ such that $k \in L_{\Omega} * L_{Y}$ can be represented as $L_{Z}$ for an appropriately chosen $Z \subseteq X$; sometimes-at least when $Y$ is open-we can describe $Z$ in a simple form merely in terms of $\Omega$ and $Y$. Now let us turn the question around. Suppose we are given once again measurable sets $\Omega \subseteq \Gamma$ and $Y \subseteq X$, but this time we are interested in the collection of all $k \in L_{X}$ such that $L_{\Omega} * k \subseteq L_{Y}$. We will show that this collection forms an $L_{Z}$ and we will describe $Z$ in terms of $\Omega$ and $Y$.

First we have a preliminary proposition, a kind of counterpart to the Decomposition Theorem.

3.14. TheOREM. Let $K$ be an absolutely continuous module over $L_{\Gamma}$. Also let $\Omega \subseteq \Gamma$ be measurable and let $B$ be a closed linear subspace of $K$. Then

$$
\left\{k \in K: L_{\Omega} * k \subseteq B\right\}=\bigcap_{\sigma \in d \Omega} B_{\sigma} .
$$


Proof. Let $L_{\Omega} * k \subseteq B$ and $\sigma \in d \Omega$. Then, by Lemma 3.1, there is an approximate identity $\left(u_{i}\right)_{i \in I}$ in $L_{\Gamma}$ with $\left(u_{i}\right)^{\sigma^{-1}} \in L_{\Omega}$ for every $i \in I$. This means that $k_{\sigma^{-1}}=\lim _{i}\left(u_{i}^{\sigma^{-1}} * k\right) \in B$, so that $k \in B_{\sigma}$, which therefore holds for all $\sigma \in d \Omega$. On the other hand, let $k \in \bigcap_{\sigma \in d \Omega} B_{\sigma}$ and let $k^{*} \in K^{*}$ with the property that $k^{*}=0$ on $B$. Then $k^{*}\left(k_{\sigma^{-1}}\right)=0$ for locally almost all $\sigma \in \Omega$. Thus, for any $f \in L_{\Omega}, k^{*}(f * k)$ $=\int_{\Gamma} f(\sigma) k^{*}\left(k_{\sigma^{-1}}\right) d \sigma=0$. Since this is true for all $k^{*} \in K^{*}$ which vanish on $B$, we obtain $f * k \in B$. Consequently, $L_{\Omega} * k \subseteq B$.

We turn to more concrete examples.

3.15. Theorem. Let $\Omega \subseteq \Gamma$ and $Y \subseteq X$ be measurable, $A=\{x \in X: \sigma x \in Y$ for locally almost all $\sigma \in \Omega\}$. Then $A$ is measurable and $L_{A}^{p}=\left\{k \in L_{X}^{p}: L_{\Omega} * k \subseteq L_{Y}^{p}\right\}$ for every $p \in[1, \infty]$.

Proof. First let $p=1$. Inasmuch as $\bigcap_{\sigma \in d \Omega}\left(L_{Y}\right)_{\sigma}=\bigcap_{\sigma \in d \Omega} L_{\sigma^{-1} Y}$ is a module over $L_{X}^{\infty}$ under pointwise multiplication, the space $\left\{k \in L_{X}: L_{\Omega} * k \subseteq L_{Y}\right\}$ is of the form $L_{z}$, by Theorem 3.9. All we need to prove, then, is that if $D$ is compact in $X$, then $D \subseteq Z$ a.e. if and only if $D \subseteq A$ a.e. Therefore let $D \subseteq A$ a.e. This means that $\{\sigma \in \Omega: \sigma x \in X \backslash Y\}$ is locally null for almost all $x \in D$, so that for any compact subset $\Phi$ of $\Omega$,

$$
\begin{aligned}
0 & =\int_{X} \xi_{D}(x) \int_{\Gamma} \xi_{\Phi}(\sigma) \xi_{X \mid Y}(\sigma x) d \sigma d x \\
& =\int_{\Gamma} \xi_{\Phi}(\sigma) \int_{X} \xi_{D}(x) \xi_{X \mid Y}(\sigma x) d x d \sigma \\
& =\int_{\Gamma} \xi_{\Phi}(\sigma) m\left(D \cap \sigma^{-1}(X \backslash Y)\right) d \sigma .
\end{aligned}
$$

Therefore $m_{X}\left(D \cap \sigma^{-1}(X \backslash Y)\right)=0$ for locally almost all $\sigma \in \Omega$. By the quasiinvariance of $m_{X}, 0=m_{X}(\sigma D \cap(X \backslash Y))=\xi_{X \mid Y}\left(\xi_{\sigma D}\right)=\xi_{X \mid Y}\left[\left(\xi_{D}\right)_{\sigma}^{-1}\right]$ for locally almost all $\sigma \in \Omega$. Now $\left(\xi_{D}\right)_{\sigma^{-1}}$ depends continuously on $\sigma$, so that $\xi_{X \mid Y}\left[\left(\xi_{D}\right)_{\sigma^{-1}}\right]=0$ for all $\sigma \in d \Omega$. In other words $\sigma \in d \Omega$ implies that $\sigma D \subseteq Y$ l.a.e., so that $\xi_{D} \in \bigcap_{\sigma \in d \Omega} L_{\sigma^{-1} Y}$ and $D \subseteq Z$ a.e. Since the procedure is reversible, $D \subseteq Z$ a.e. implies that $D \subseteq A$ a.e., and the case $p=1$ is completed.

Next, let $p \in(1, \infty)$. By Theorem 3.14, $\left\{k \in L_{X}^{p}: L_{\Omega} * k \subseteq L_{Y}^{p}\right\}=\bigcap_{\sigma \in d \Omega} L_{\sigma}^{p-1} Y$, which in turn is $\left\{k \in L_{X}^{p}:|k|^{p} \in \bigcap_{\sigma \in d \Omega} L_{\sigma^{-1} Y}\right\}$. However, $\bigcap_{\sigma \in d \Omega} L_{\sigma^{-1} Y}=L_{A}$ by the first part of the proof, so the collection is none other than $L_{A}^{p}$. For $p=\infty$, we obtain $\left\{k \in L^{\infty}(X): L_{\Omega} * k \subseteq L_{Y}^{\infty}\right\}=\bigcap_{\sigma \in d \Omega} L_{\sigma^{-1} Y}^{\infty}=\left\{k \in L^{\infty}(X): k \xi_{D} \in \bigcap_{\sigma \in d \Omega} L_{\sigma}^{p}{ }^{-1} Y\right.$ for every compact $D \subseteq X\}=\left\{k \in L^{\infty}(X): k \xi_{D} \in L_{A}\right.$ for every compact $\left.D \subseteq X\right\}=L_{A}^{\infty}$.

4. The orbit topology and its consequences. From now on $\Gamma$ is a group of homeomorphisms of $X$ on which there is a quasi-invariant measure $m_{X}$. We borrow the next two theorems from [4]. After that we shall assume throughout the rest of the paper that $\Gamma$ and $X$ satisfy any and hence all the conditions of Theorem 4.1.

4.1. THEOREM. The following three conditions are equivalent:

(a) If $D \subseteq X$ is compact and $m_{X}(D)=0$, and if $a \in X$, then $\sigma a \notin D$ for locally almost every $\sigma \in \Gamma$. 
(b) If $\Phi \subseteq \Gamma$ is a Borel set with positive measure, then for all $a \in X$, Фa has positive outer measure.

(c) Let $\Gamma_{0}$ be an open $\sigma$-compact subgroup of $\Gamma$. Then for every $a \in X, \Gamma_{0} a$ is measurable and $m_{X}\left(\Gamma_{0} a\right)>0$.

(We note that such $\Gamma_{0}$ exists in every case: every compact neighborhood of 1 generates an open $\sigma$-compact group.) The proof of this theorem is contained in 5.6 and 5.7 of [4].

The conditions of Theorem 4.1 are satisfied if $X$ is a factor space of $\Gamma$ and the action of $\Gamma$ on $X$ is the natural one [4, remark made immediately before 5.8].

4.2. TheOrem. Let $a \in X$. For $\sigma \in \Gamma$ let $\pi_{a}(\sigma)=\sigma a$. Then $\pi_{a}$ is a continuous open map of $\Gamma$ onto $\Gamma a$. Further, $\Gamma a$ is the intersection of a closed and open set in $X$, and therefore is measurable (see 5.10 of [4]).

Next we let $\mathscr{L}^{1}(X)$ be the collection of functions on $X$ which are integrable ( $L_{X}$ still denotes the space of all classes of integrable functions that are a.e. equal), and similarly for $\mathscr{L}^{\infty}$. For $f \in \mathscr{L}^{1}(\Gamma)$ and $k \in \mathscr{L}^{\infty}(X)$ we put

$$
(f \circ k)(x)=\int_{\Gamma} f(\sigma) k\left(\sigma^{-1} x\right) d \sigma
$$

for all $x \in X$ for which the integral exists. Manifestly, if $f_{1}, f_{2} \in \mathscr{L}^{1}(\Gamma)$ and $f_{1}=f_{2}$ a.e., then $f_{1} \circ k=f_{2} \circ k$.

4.3. THEOREM. The following condition is equivalent to each of those stated in Theorem 4.1:

If $f \in \mathscr{L}^{1}(\Gamma)$ and $k_{1}, k_{2} \in \mathscr{L}^{\infty}(X)$ and if $k_{1}=k_{2}$ l.a.e., then $f \circ k_{1}$ and $f \circ k_{2}$ are defined everywhere on $X$, and $f \circ k_{1}=f \circ k_{2}$.

Proof. First we show that the above condition implies (a) of Theorem 4.1. Let $D$ be compact in $X$ with $m_{X}(D)=0$, and let $a \in X$. By our assumption,

$$
0=\left(f \circ \xi_{D}\right)(a)=\int_{\Gamma} f(\sigma) \xi_{D}\left(\sigma^{-1} a\right) d \sigma \quad \text { for all } f \in \mathscr{L}^{1}(\Gamma) .
$$

But this just says that $\sigma^{-1} a \notin D$ for locally almost all $\sigma \in \Gamma$, which is (a). Now we utilize (b) of Theorem 4.1 to prove the above condition. We need only take $k \in \mathscr{L}^{\infty}(X)$ such that $k=0$ 1.a.e., and show that for each $f \in \mathscr{L}^{1}(\Gamma)$ and $a \in X$, $(f \circ k)(a)=0$. By the contrapositive of (b), $k\left(\sigma^{-1} a\right)=0$ 1.a.e. in $\Gamma$. Thus $(f \circ k)(a)=\int_{\Gamma} f(\sigma) k\left(\sigma^{-1} a\right) d \sigma=0$.

The importance of Theorem 4.3 is that we may consider $f \circ k$ as defined for $f \in L_{\Gamma}, k \in L_{X}^{\infty}$, rather than for $f \in \mathscr{L}^{1}(\Gamma), k \in \mathscr{L}^{\infty}(X)$-providing the pair $\Gamma$ and $X$ satisfy the conditions of Theorem 4.1. We define $L_{\Gamma} \circ L_{X}^{\infty}$ as $\left\{f \circ k: f \in L_{\Gamma}, k \in L_{X}^{\infty}\right\}$.

Let $\Omega$ be a measurable subset of $\Gamma$ and $Y$ a measurable subset of $X$. By Theorem 3.9 we know there exists a measurable subset $Z$ of $X$ such that $L_{\Omega} * L_{Y}=L_{Z}$. In order to give an explicit form to a $Z$ for which $L_{\Omega} * L_{Y}=L_{Z}$, we make use of two 
entities, one a topology on $X$ which is customarily different from the given topology, and the second a set $T$ in $X$ which is directly related to the convolution and which we will show is locally the same as $Z$. Hereafter we designate the original topology on $X$ by $\mathscr{T}$. We begin by discussing the new topology on $X$, which we denote by $\mathcal{O}$. It is described in terms of the orbits $\Gamma x$ of the elements $x$ in $X$.

4.4. Definition. A basis for the topology $\mathcal{O}$ consists of sets of the form $\{\Phi x: \Phi$ open in $\Gamma, x \in X\}$. We call this topology the orbit topology (on $X$ ).

The fact that the collection just described forms a basis for a bona fide topology on $X$ is immediate. $\mathcal{O}$ is a natural analog of $\mathscr{T}$, in the sense that $\mathscr{T}$ is generated by the sets of the form $\{\sigma U: \sigma \in \Gamma, U$ open in $X\}$. However, the two topologies need not be identical. For an example, let $\boldsymbol{R}$ be the additive group of reals with the usual topology, and let $\Gamma=\boldsymbol{R}$ and $X=\boldsymbol{R} \cup\{\infty\}$ the one-point compactification of $\boldsymbol{R}$. Let $\delta_{\infty}$ be the point mass at $\infty$ and let $m_{X}$ be defined by

$$
m_{X}(Y)=m(R \cap Y)+\delta_{\infty}(Y), \text { for any Borel set } Y \text { in } X .
$$

Detine the action of $\Gamma$ on $X$ by

$(\sigma, x) \rightarrow x+\sigma, x \in R, \sigma \in \Gamma$, $(\sigma, \infty) \rightarrow \infty, \sigma \in \Gamma$.

This system satisfies the conditions mentioned in Theorem 4.1. The set $\{\infty\}$ is not open in $\mathscr{T}$, while $\{\infty\}=\Gamma\{\infty\}$ is both closed and open in $\mathcal{O}$.

Let us nail down a few of the properties of $\mathcal{O}$.

4.5. Lemma. (i) $\mathcal{O}$ is at least as fine as $\mathscr{T}$.

(ii) For each $x \in X, \mathcal{O}$ coincides with $\mathscr{T}$ on $\Gamma x$.

Proof. (i) If $U$ is a neighborhood of $a$ in $\mathscr{T}$, then there is an open set $\Phi \subseteq \Gamma$ such that $\Phi a \subseteq U$, since the map $(\sigma, x) \rightarrow \sigma x$ is jointly continuous. But $\Phi a$ is a $\mathcal{O}$ neighborhood of $a$. To prove (ii) let $\Phi$ be open in $\Gamma$, and $a \in X$. By Theorem 4.2, the map $\pi_{a}: \Gamma \rightarrow \Gamma a$ is $\mathscr{T}$-open, so we are done.

Lemma 4.5 yields two characterizations of $\mathcal{O}$. We assume henceforth that $\Gamma_{0}$ is an open sigma-compact subgroup of $\Gamma$.

(i) $U \subseteq X$ is $\mathcal{O}$-open if and only if for each $x \in X$, the set $\{\sigma \in \Gamma: \sigma x \in U\}$ is open in $\Gamma$.

(ii) $U \subseteq X$ is $\mathcal{O}$-open if and only if for each $x \in X, U \cap \Gamma_{0} x$ is relatively open in $\Gamma_{0} x$.

We push on with the characteristics of $\mathcal{O}$.

4.6. Lemma. (i) Let $U \subseteq X$ be $\mathscr{T}$-open and $a \in X$. Then either $U \cap \Gamma_{0} a=\varnothing$ or $m_{X}\left(U \cap \Gamma_{0} a\right)>0$.

(ii) Every set with finite outer measure in $X$ intersects only countably many $\Gamma_{0}$-orbits.

(iii) Every $\mathscr{T}$-compact set is a union of countably many $\mathcal{O}$-compact sets.

Proof. (i) Assume that $U \cap \Gamma_{0} a \neq \varnothing$. Since $\Gamma_{0}$ is sigma-compact and $(\sigma, x) \rightarrow \sigma x$ is continuous, $\Gamma_{0} a$ is sigma-compact; thus $U \cap \Gamma_{0} a$ is measurable. There is an 
open set $\Phi \in \Gamma_{0}$ such that $\Phi a \subseteq U$. Thus $\Phi a \subseteq\left(U \cap \Gamma_{0} a\right)$. Using Theorem 4.1(c), we have $0<m_{X}(\Phi a) \leqq m_{X}\left(U \cap \Gamma_{0} a\right)$.

To prove (ii), let $Y \subseteq X$ be of finite outer measure. Since $X$ is locally compact there is an open set $U$ such that $U \supseteq Y$ and $m_{X}(U)<\infty$. By (i), $m_{X}\left(U \cap \Gamma_{0} x\right)>0$ for every $x \in X$ such that $U \cap \Gamma_{0} x \neq \varnothing$. Then there can be only countably many orbits $\Gamma_{0} x$ with $U \cap \Gamma_{0} x \neq \varnothing$, and (ii) is proved.

For (iii), let $D \subseteq X$ be $\mathscr{T}$-compact. By (ii) there exists a sequence $\left(x_{n}\right)$ in $X$ such that $D=\bigcup_{n=1}^{\infty}\left(D \cap \Gamma_{0} x_{n}\right)$. But $\Gamma_{0}$ is by definition sigma-compact in $\Gamma$, whence $\Gamma_{0} x_{n}$ is sigma-compact in $X$, so that $D \cap \Gamma_{0} x_{n}$ is sigma-compact in $(X, \mathscr{T})$. Inasmuch as the original and $\mathcal{O}$-topology coincide on each $\Gamma x_{n}$, (iii) is also proved.

By Lemma 4.6(iii), $\mathscr{T}$ and $\mathcal{O}$ have the same sigma-compact sets so the notion of 1.a.e. is the same with respect to both topologies.

In the next two propositions we show that $\mathcal{O}$ is a legitimate topology to work with, with respect to $\Gamma$ and $m_{x}$.

4.7. Lemma. Every $\mathcal{O}$-open set $U$ is $m_{X}$-measurable, and if $U \neq \varnothing$, then $m_{X}(U)>0$.

Proof. Let $U$ be $\mathcal{O}$-open in $X$. By 11.31 of [5] we only need to show that $U \cap V$ is measurable for every $V \subseteq X$ that is open in the $\mathscr{T}$-topology and has finite measure. Since $V$ is automatically $\mathcal{O}$-open, we may assume that $U=U \cap V$. At least we know that $U$ has finite outer measure. Now we show it is measurable. By Lemma 4.6(ii), there is a sequence $x_{1}, x_{2}, \ldots$ such that $U=\bigcup_{n=1}^{\infty}\left(U \cap \Gamma_{0} x_{n}\right)$. Since $U$ is $\mathcal{O}$-open and $\mathscr{T}$ and $\mathscr{O}$ coincide on $\Gamma_{0} x$, for each $n$ there is an $\mathscr{T}$-open $W_{n} \subseteq X$ such that $U \cap \Gamma_{0} x_{n}=W_{n} \cap \Gamma_{0} x_{n}$. Then $U=\bigcup_{n=1}^{\infty}\left(W_{n} \cap \Gamma_{0} x_{n}\right)$, which is measurable. To show that if $U \neq \varnothing$ then $m_{X}(U)>0$, we let $x \in U$ and find a $\mathscr{T}$-open $V$ in $X$ such that $x \in V \cap \Gamma_{0} x=U \cap \Gamma_{0} x$. By (i) of Lemma 4.6, $m_{X}(U) \geqq m_{X}\left(V \cap \Gamma_{0} x\right)>0$.

We remark that Lemma 4.7 says that if $\Phi$ is open in $\Gamma$ and $x \in X$, then $m_{X}(\Phi x)>0$.

4.8. THEOREM. $(X, \mathcal{O})$ is locally compact, and $\Gamma$ acts as a transformation group on $(X, \mathcal{O})$. Furthermore, $m_{X}$ is a quasi-invariant Radon measure on $(X, \mathcal{O})$.

Proof. Since each $\Gamma x$ is, in the $\mathscr{T}$-topology, the intersection of an open and a closed subset of $X$ by Theorem 4.2, it is locally compact. But $\Gamma x$ is $\mathcal{O}$-open, so $\mathcal{O}$ is a locally compact topology for $X$. To show that $\Gamma$ acts as a transformation group on $(X, \mathcal{O})$, we note that $\sigma \in \Gamma$ implies $\sigma$ is an $\mathcal{O}$-homeomorphism. Now we show that $(\sigma, x) \rightarrow \sigma x$ is $\mathcal{O}$-jointly continuous. Let $(\sigma, a)$ be fixed in $\Gamma \times X$ and $U$ an $\mathcal{O}$ neighborhood of $\sigma a$. Straight from the definition of $\mathcal{O}$, there is a neighborhood $\Phi$ of 1 in $\Gamma$ such that $\sigma(\Phi \Phi) a \subseteq U$. Then $\sigma \Phi, \Phi a$ are neighborhoods of $\sigma, a$ respectively, and $(\sigma \Phi)(\Phi a) \subseteq U$.

Finally we prove that $m_{X}$ is a Radon measure on $(X, \mathcal{O})$. We denote by $m^{*}$ and $m_{*}$ the outer and inner measure respectively of $m_{X}$. Let $Y$ be any subset of $X$. Since every $\mathscr{T}$-open set is $\mathcal{O}$-open,

$$
\begin{aligned}
m^{*}(Y) & =\inf \left\{m_{X}(Z): Y \subseteq Z, Z \mathscr{T} \text {-open }\right\} \\
& \geqq \inf \left\{m^{*}(Z): Y \subseteq Z, Z \mathcal{O} \text {-open }\right\} \geqq m^{*}(Y) .
\end{aligned}
$$


By Lemma 4.7 , if $Z$ is $\mathcal{O}$-open, then it is measurable, so we may write $m_{X}(Z)$ instead of $m^{*}(Z)$. Thus $m^{*}(Y)=\inf \{m(Z): Y \subseteq Z, Z \mathcal{O}$-open $\}$. On the other hand, every $\mathcal{O}$-compact set in $X$ is $\mathscr{T}$-compact, while every $\mathscr{T}$-compact set is the union of an increasing sequence of $\mathcal{O}$-compact sets. Therefore for any $Y \subseteq X$, $m_{*}(Y)=\sup \{m(D): D \subseteq X$ and $D \mathcal{O}$-compact $\}$. The formulas for $m^{*}$ and $m_{*}$ yield $m_{X}$ - the same as $m_{X}$ with respect to the original topology - a Radon measure with respect to $\mathcal{O}$. Thus $m_{X}$ is quasi-invariant with respect to $\mathcal{O}$.

4.9. Lemma. (i) $\mathcal{O}$ is the weakest topology on $X$ for which $L_{\Gamma} \circ L_{X}^{\infty} \subseteq C(X)$.

(ii) $\mathcal{O}=\mathscr{T}$ if and only if for each compact $\Psi \subseteq \Gamma$ and each relatively $\mathscr{T}$-compact measurable $D \subseteq X$, the map $x \rightarrow m\left\{\sigma \in \Psi: \sigma^{-1} x \in D\right\}$ is continuous.

Proof. Let $a \in X$ and let $f \in L_{\Gamma}, k \in L_{X}^{\infty}$. Then for each $\sigma \in \Gamma$,

$$
(f \circ k)(\sigma a)=\int_{\Gamma} f(\tau) k\left(\tau^{-1} \sigma a\right) d \tau=\left(f_{\sigma} \circ k\right)(a) .
$$

But the shift in $L_{\Gamma}$ is continuous, and $\pi_{a}: \Gamma \rightarrow \Gamma a$ is an open map, so on the orbit $\Gamma a, f \circ k$ is $\mathcal{O}$-continuous. Since $a$ is arbitrary and $\Gamma a$ is $\mathcal{O}$-open, $f \circ k$ is $\mathcal{O}$-continuous and for the topology $\mathcal{O}$ on $X, L_{\Gamma} \circ L_{X}^{\infty} \subseteq C(X)$. Conversely, let $U$ be an $\mathcal{O}$-neighborhood of $a \in X$. We shall find $f \in L_{\Gamma}$ and $k \in L_{X}^{\infty}$ such that $f \circ k(a) \neq 0$ and $f \circ k=0$ on $X \backslash U$; this proves that $L_{\Gamma} \circ L_{X}^{\infty} \subseteq C(X)$ can not hold for any topology that is strictly weaker than $\mathcal{O}$. Since $\Gamma$ acts as a transformation group on $(X, \mathcal{O})$ there is a neighborhood $\Phi$ of $1 \in \Gamma$ such that $m(\Phi)<\infty$ and $\Phi a \subseteq U$. Then there is an $\mathcal{O}$-open, hence measurable, set $Z \subseteq X$ such that $a \in Z$ and $\Phi Z \subseteq U$. Then $\xi_{\Phi} \in L_{\Gamma}$ and $\xi_{Z} \in L_{X}^{\infty}$. Clearly $\xi_{\Phi} \circ \xi_{Z}=0$ on $X \mid U$, and $\xi_{\Phi} \circ \xi_{Z}(a)=m\left\{\sigma \in \Phi: \sigma^{-1} a \in Z\right\}>0,\left\{\sigma \in \Phi: \sigma^{-1} a \in Z\right\}$ being an open neighborhood of 1 in $\Gamma$.

Now we prove (ii). We note that if $\Psi \subseteq \Gamma$ is compact and $D \subseteq X$ is relatively compact, then $\xi_{\Psi} \circ \xi_{D}(x)=m\left\{\sigma \in \Psi: \sigma^{-1} x \in D\right\}$, so that the assumption $L_{\Gamma} \circ L_{X}^{\infty} \subseteq C(X)$ implies the condition. To prove the converse it suffices to consider any compact set $\Psi \subseteq \Gamma$ and any measurable set $Y \subseteq X$ and show that $\xi_{\Psi} \circ \xi_{Y}$ is continous at any given $a \in X$. To that end, we note that $\Psi^{-1} a$ is compact in $X$, so that there is a $D$, compact in $X$, whose interior contains $\Psi^{-1} a$. Let $W$ be a neighborhood of $a$ such that $\Psi^{-1} W \subseteq D$, and let $Q=D \cap Y$. If $y \in W$, then

$$
\begin{aligned}
\xi_{\Psi} \circ \xi_{Q}(y) & =m\left\{\sigma \in \Psi: \sigma^{-1} y \in Q\right\}=m\left\{\sigma \in \Psi: \sigma^{-1} y \in Q \cap \Psi^{-1} W\right\} \\
& =m\left\{\sigma \in \Psi: \sigma^{-1} y \in Y \cap \Psi^{-1} W\right\}=m\left\{\sigma \in \Psi: \sigma^{-1} y \in Y\right\}=\left(\xi_{\Psi} \circ \xi_{Y}\right)(y) .
\end{aligned}
$$

Consequently, on $W, \xi_{\Psi} \circ \xi_{Y}=\xi_{\Psi} \circ \xi_{Q}$. Since $Q$ is relatively compact and $\Psi$ is compact, the hypothesis that $x \rightarrow\left\{\sigma \in \Psi: \sigma^{-1} x \in Q\right\}$ be continuous merely says that $\xi_{\Psi} \circ \xi_{Q}$ is continuous on $X$, and hence on $W$. Therefore, $\xi_{\Psi} \circ \xi_{Y}$ is continuous on $W$, and hence at $a$. Since $a$ was arbitrary, $\xi_{\Psi} \circ \xi_{Y}$ is continuous, as desired.

In the proofs of the theorems which we are leading up to, we employ the $\mathcal{O}$ continuity of functions $f \circ k$. What will especially interest us are the sets where various $f \circ k$ are nonzero, which we know to be $\mathcal{O}$-open via Lemma 4.9(i). Let $\Omega \subseteq \Gamma$ and $Y \subseteq X$ be measurable. 
4.10. Definition. Let $T=\{x \in X$ : there exist $\Phi \subseteq \Gamma, D \subseteq X$ each with finite measure, such that $\Phi \subseteq \Omega$ l.a.e. and $D \subseteq Y$ l.a.e., and such that $\left.\left(\xi_{\Phi} \circ \xi_{D}\right)(x)>0\right\}$.

We begin discussing $T$ by proving a lemma connecting $\circ$ and $*$.

4.11. Lemma. (i) $x \in T$ if and only if there exist compact sets $\Phi^{\prime} \subseteq \Omega$ and $D^{\prime} \subseteq X$ such that $\left(\xi_{\Phi^{\prime}} \circ \xi_{D^{\prime}}\right)(y)>0$.

(ii) Let $\Phi \subseteq \Gamma$ and $D \subseteq X$ be compact. Then

$$
\begin{array}{ll}
\xi_{\Phi} * \xi_{D}>0 & \text { l.a.e. on }\left\{x \in X:\left(\xi_{\Phi} \circ \xi_{D}\right)(x)>0\right\} \\
\xi_{\Phi} * \xi_{D}=0 & \text { l.a.e. on }\left\{x \in X:\left(\xi_{\Phi} \circ \xi_{D}\right)(x)=0\right\} .
\end{array}
$$

Proof. (i) The "if" part is trivial. Now assume $x \in T$. Let $\Phi, D$ be as in the definition of $T$. By Theorem 4.3 and finite measures of $\Phi$ and $D$ we may assume $\Phi \subseteq \Omega$ and $D \subseteq Y$, and also that $\Phi, D$ are sigma-compact. Let $\Phi_{1}, \Phi_{2}, \ldots$ and $D_{1}, D_{2}, \ldots$ be compact sets such that $\Phi=\bigcup_{k=1}^{\infty} \Phi_{k}, D=\bigcup_{n=1}^{\infty} D_{n}$. Then

$$
0<\left(\xi_{\Phi} \circ \xi_{D}\right)(x)=m\left(\left\{\sigma \in \Phi: \sigma^{-1} x \in D\right\}\right)=m\left(\bigcup_{k, n}\left\{\sigma \in \Phi_{k}: \sigma^{-1} x \in D_{n}\right\}\right) .
$$

It follows that for some $k$ and $n, m\left(\left\{\sigma \in \Phi_{k}: \sigma^{-1} x \in D_{n}\right\}\right)>0$. Put $\Phi^{\prime}=\Phi_{k}, D^{\prime}=D_{n}$, and we have $\left(\xi_{\Phi^{\prime}} \circ \xi_{D^{\prime}}\right)(x)>0$.

(ii) For $f \in L_{\Gamma}$ and $k \in L_{X}^{\infty}$, we have (see $\left.\S 2\right)$ that $f * k(x)=\int_{\Gamma} f(\sigma) k\left(\sigma^{-1} x\right) J\left(\sigma^{-1}, x\right) d \sigma$ for locally almost every $x$. It follows that for l.a.e. $x,\left(\xi_{\Phi} * \xi_{D}\right)(x)=0$ if and only if $\xi_{\Phi}(\sigma) \xi_{D}\left(\sigma^{-1} x\right)=0$ 1.a.e. on $\Gamma$. This proves (ii).

4.12. LEMMA. (i) $T$ is $\mathcal{O}$-open, and hence measurable.

(ii) $T \subseteq \Omega Y$.

(iii) $L_{\Omega} * L_{Y} \subseteq L_{T}$.

Proof. Since each $f \circ k$ is $\mathcal{O}$-continuous, $T$ is $\mathcal{O}$-open, and by Lemma $4.7, T$ is measurable, so we have (i) sewed up. To prove (ii), we note that if $x \notin \Omega Y$, then for any $\Phi \subseteq \Omega$ and $Z \subseteq Y$ with finite measure, $0=\int_{\Gamma} \xi_{\Phi}(\sigma) \xi_{Z}\left(\sigma^{-1} x\right) d \sigma=\xi_{\Phi} \circ \xi_{Z}(x)$, so $x \notin T$. Finally, we prove that $L_{\Omega} * L_{Y} \subseteq L_{T}$. Since for any compact sets $\Psi \subseteq \Omega$ and $D \subseteq Y, \xi_{\Psi} * \xi_{D}$ is 0 a.e. on $X \mid T$ (see Lemma 4.11(ii)), we have $\xi_{\Psi} * \xi_{D} \in L_{T}$. But $L_{\Omega} * L_{Y}$ is generated by convolutions of such functions. Thus $L_{\Omega} * L_{Y} \subseteq L_{T}$.

Next we see how $T$ is affected by the operators $d$ and $i$ (see the beginning of $\S 3$ ). Of course $d$ and $i$ depend upon the topology of $X$. If we consider $d$ and $i$ with respect to the $\mathcal{O}$-topology, we will denote them by $d_{\mathcal{O}}$ and $i_{\mathcal{O}}$. In $\mathscr{T}$ we will just use the plain $d$ and $i$. Now we produce a lemma closely associated with Corollary 3.5.

4.13. LemMA. (i) $d \Omega d_{\mathscr{O}} Y$ is contained in the $\mathcal{O}$-closure of $T$.

(ii) $d \Omega i_{\mathcal{O}} Y \subseteq T$.

Proof. To prove (i), note that $L_{\Omega} * L_{Y} \subseteq L_{T}$. Hence $(d \Omega)\left(d_{\mathcal{O}} Y\right) \subseteq d_{\mathcal{O}} T$ by Corollary 3.5(ii). Further, $d_{\mathcal{O}} T$ lies in the $\mathcal{O}$-closure of $T$. Now we prove (ii). Let $\sigma \in d \Omega$ and $a \in i_{\mathcal{O}} Y$ and let $D$ be a $\mathcal{O}$-compact neighborhood of $a$ such that $m_{X}(D \backslash Y)=0$. In 
addition let $\Phi$ be a neighborhood of the identity in $\Gamma$ of finite measure and such that $\Phi^{-1} a \subseteq D$. Inasmuch as $\tau \in \sigma \Phi \cap \Omega$ implies that $\tau^{-1} \sigma a \in \Phi^{-1} a \subseteq D$, we have the following inequalities:

$$
\begin{aligned}
\left(\xi_{\sigma \Phi \cap \Omega} \circ \xi_{D \cap Y}\right)(\sigma a) & =\int_{\Gamma} \xi_{\sigma \Phi \cap \Omega}(\tau) \xi_{D \cap Y}\left(\tau^{-1} \sigma a\right) d \tau \\
& =\int_{\Gamma} \xi_{\sigma \Phi \cap \Omega}(\tau) \xi_{D}\left(\tau^{-1} \sigma a\right) d \tau \\
& \geqq \int_{\Gamma} \xi_{\sigma \Phi \cap \Omega}(\tau) d \tau \\
& >0 \quad(\text { since } \sigma \in d \Omega)
\end{aligned}
$$

so that $\sigma a \in T$.

4.14. THEOREM. $L_{\Omega} * L_{Y}=L_{T}$.

Proof. In the first place, $L_{\Omega} * L_{Y} \subseteq L_{T}$ by Lemma 4.12(iii). To prove the reverse inclusion, we refer to the measurable $Z \subseteq X$ for which $L_{\Omega} * L_{Y}=L_{Z}$ (Theorem 3.9). Let us prove that $T \subseteq i_{\mathcal{O}} Z$. To begin, let $a \in T$. Then there are compact sets $\Phi \subseteq \Omega$ and $D \subseteq Y$ such that $\left(\xi_{\Phi} \circ \xi_{D}\right)(a)>0$. If $U=\left\{x \in X:\left(\xi_{\Phi} \circ \xi_{D}\right)(x)>0\right\}$, then $U$ is an $\mathcal{O}$-neighborhood of $a$, and since $\xi_{\Phi} \circ \xi_{D}>0$ on $U$, we must have $U \subseteq Z$ 1.a.e. Thus $a \in i_{\mathcal{O}} Z$. Consequently $T \subseteq i_{\mathcal{O}} Z \subseteq Z$ 1.a.e., so that $L_{T} \subseteq L_{Z}=L_{\Omega} * L_{Y}$.

5. Vanishing modules. The central problem we tackle in this section is the relationship between the two expressions $\Omega Y \subseteq Y$ and $L_{\Omega} * L_{Y} \subseteq L_{Y}$. They look as though they ought to be related; perhaps they are even equivalent. However, even at the outset trouble looms, because $\Omega Y \subseteq Y$ is set-theoretic and $L_{\Omega} * L_{Y} \subseteq L_{Y}$ is measure-theoretic. Nevertheless, let us work on the problem and see what we can harvest.

5.1. THEOREM. If $\Omega Y \subseteq Y$, then $L_{\Omega} * L_{Y} \subseteq L_{Y}$.

Proof. Straightforward from the convolution formula (see §2).

That was simple. However, the converse of Theorem 5.1 is by no means so simple, even if $X=\Gamma$. A conjecture arose in [10] that if $\Omega \subseteq \Gamma$ is relatively sigma-compact in $\Gamma$, and if $L_{\Omega} * L_{\Omega} \subseteq L_{\Omega}$, then there exists an $\Omega^{\prime} \subseteq \Gamma$ such that $\Omega^{\prime}=\Omega$ a.e. and $\Omega^{\prime} \Omega^{\prime} \subseteq \Omega^{\prime}$. Finally this was proved in [6]. For $\Omega$ which is not relatively sigmacompact the answer is yet unknown, so far as we can determine. Consequently the extension from subalgebras of $L_{\Gamma}$ to submodules of $L_{X}$ can be expected to bring great difficulty.

A subalgebra $L_{\Omega}$ of $L_{\Gamma}$ with the property that $L_{\Omega} * L_{\Omega} \subseteq L_{\Omega}$ has been christened a vanishing algebra [10], since it vanishes outside $\Omega$. A subspace $L_{Y}$ of $L_{X}$ with the property that for a specific $L_{\Omega}$ in $L_{\Gamma}$ one has $L_{\Omega} * L_{Y} \subseteq L_{Y}$ might be called a vanishing submodule of $L_{X}$. In our proposition we refer to symbolic notation for clarity and simplicity. 
5.2. TheOREM. Let $\Omega \subseteq \Gamma$ and $Y \subseteq X$ be measurable relatively sigma-compact sets. Then there exist $\Omega_{0} \subseteq \Gamma$ and $Y_{0} \subseteq X$ such that $\Omega_{0}=\Omega$ a.e., and $Y_{0}=Y$ a.e., and such that $\Omega_{0} Y_{0}=T$.

Proof. For the present we assume that $\Omega$ and $Y$ are relatively compact. For each $x \in X$, let $Y_{x}=\left\{\sigma \in \Gamma: \sigma^{-1} x \in Y\right\}$. If $\Phi$ is any compact neighborhood of $1 \in \Gamma$, define $f_{\Phi, Y}$ and $g_{\Phi, \Omega}$ by

$$
\begin{array}{lll}
f_{\Phi, \mathrm{Y}}(x)=m\left(Y_{x} \cap \Phi\right) / m(\Phi), & & x \in X, \\
g_{\Phi, \Omega}(\sigma)=m\left(\sigma^{-1} \Omega \cap \Phi\right) / m(\Phi), & & \sigma \in \Gamma .
\end{array}
$$

First we note that $f_{\Phi, Y}$ and $g_{\Phi, \Omega}$ are measurable. Given any measurable set $Z \subseteq X$, we have

$$
\begin{aligned}
\left|\int_{Z}\left[\xi_{Y}(x)-f_{\Phi, Y}(x)\right] d x\right| & =\left|\frac{1}{m(\Phi)} \int_{Z} \int_{\Phi}\left[\xi_{Y}(x)-\xi_{Y_{x}}(\sigma)\right] d \sigma d x\right| \\
& \leqq \frac{1}{m(\Phi)} \int_{\Phi} d \sigma \int_{Z}\left|\xi_{Y}(x)-\xi_{\sigma Y}(x)\right| d x \\
& =\frac{1}{m(\Phi)} \int_{\Phi} m_{X}(Z \cap(Y \Delta \sigma Y)) d \sigma \\
& \leqq \sup _{\sigma \in \Phi} m_{X}(Y \Delta \sigma Y),
\end{aligned}
$$

where $\Delta$ is used to denote the symmetric difference of sets. Thus $\left\|\xi_{Y}-f_{\Phi, Y}\right\|_{1}$ $\leqq 2 \sup _{\sigma \in \Phi} m_{X}(Y \Delta \sigma Y)$. Similarly, $\left\|\xi_{\Omega}-g_{\Phi, \Omega}\right\|_{1} \leqq 2 \sup _{\sigma \in \Phi} m\left(\Omega \Delta \Omega \sigma^{-1}\right)$. Since $\Omega$ and $Y$ are relatively compact, the functions $\sigma \rightarrow m_{X}(Y \Delta \sigma Y)$ and $\sigma \rightarrow m\left(\Omega \Delta \Omega \sigma^{-1}\right)$ are continuous at 1 by Corollary 3.6 of [4]. Hence, for every $\varepsilon>0$ there exists a neighborhood $\Phi$ of 1 such that $\left\|\xi_{Y}-f_{\Phi, Y}\right\|_{1}<\varepsilon$ and $\left\|\xi_{\Omega}-g_{\Phi, \Omega}\right\|_{1}<\varepsilon$.

Now we drop the condition that $\Omega$ and $Y$ be relatively compact. By the hypotheses, there exist increasing sequences $\left(\Omega_{n}\right)_{n \in N}$ and $\left(Y_{n}\right)_{n \in N}$ of relatively compact sets in $\Gamma$ and $X$ respectively such that $\Omega=\bigcup_{n} \Omega_{n}$ and $Y=\bigcup_{n} Y_{n}$. For each $n$ we may choose a compact neighborhood $\Phi_{n}$ of 1 such that $\left\|\xi_{\Omega_{n}}-g_{\Phi_{n}, \Omega_{n}}\right\|_{1} \leqq n^{-2}$ and $\left\|\xi_{Y_{n}}-f_{\Phi_{n}, Y_{n}}\right\|_{1} \leqq n^{-2}$. Since $0 \leqq 1-f_{\Phi_{n}, Y} \leqq 1-f_{\Phi_{n}, Y_{n}}$, we obtain

$$
\left\|\left[1-f_{\Phi_{n}, Y}\right] \xi_{Y_{n}}\right\|_{1} \leqq\left\|\left[1-f_{\Phi_{n}, Y_{n}}\right] \xi_{Y_{n}}\right\|_{1} \leqq\left\|\xi_{Y_{n}}-f_{\Phi_{n}, Y_{n}}\right\|_{1} \leqq n^{-2} .
$$

It follows that $\lim _{n}\left\{\left[1-f_{\Phi_{n}, Y}\right] \xi_{Y_{n}}\right\}=0$ a.e., so that $\lim _{n} f_{\Phi_{n}, Y}=1$ a.e. on $Y$. In the same way one can prove that $\lim _{n} g_{\Phi_{n}, \Omega}=1$ a.e. on $\Omega$.

We have set the stage for $\Omega_{0}$ and $Y_{0}$. Let $\Omega_{0}=\left\{\sigma \in \Omega: \lim _{n} g_{\Phi_{n}, \Omega}(\sigma)=1\right\}$, and let $Y_{0}=\left\{x \in Y: \lim _{n} f_{\Phi_{n}, Y}=1\right\}$. Then $\Omega_{0}=\Omega$ a.e. and $Y_{0}=Y$ a.e. We already know from Lemma 4.12(ii) that $T \subseteq \Omega_{0} Y_{0}$. To prove the opposite inclusion, let $\sigma \in \Omega_{0}$, $y \in Y_{0}$. There is an $n$ such that $f_{\Phi_{n}, Y}(y)>\frac{1}{2}$ and $g_{\Phi_{n}, \Omega}(\sigma)>\frac{1}{2}$. If we let $\Phi=\Phi_{n}$ then $\Phi$ is a neighborhood of 1 in $\Gamma$ such that $m\left(\sigma^{-1} \Omega \cap \Phi\right)>\frac{1}{2} m(\Phi)$ and $m_{X}\left(Y_{y} \cap \Phi\right)>\frac{1}{2} m(\Phi)$. Then

$$
\begin{aligned}
m\left(\Phi \cap \sigma^{-1} \Omega \cap Y_{y}\right) & =m\left(\Phi \cap \sigma^{-1} \Omega\right)+m\left(\Phi \cap Y_{y}\right)-m\left(\Phi \cap\left(\sigma^{-1} \Omega \cup Y_{y}\right)\right) \\
& >\frac{1}{2} m(\Phi)+\frac{1}{2} m(\Phi)-m(\Phi)=0 .
\end{aligned}
$$


Thus

$$
\begin{aligned}
\xi_{\sigma \Phi \cap \Omega} \circ \xi_{\Phi^{-1} y \cap Y}(\sigma y) & =m\left(\sigma \Phi \cap \Omega \cap\left(\Phi^{-1} y \cap Y\right)_{\sigma y}\right) \\
& =m\left(\sigma \Phi \cap\left(\Phi^{-1} y\right)_{\sigma y} \cap \Omega \cap Y_{\sigma y}\right) \\
& =m\left(\sigma \Phi \cap \Omega \cap Y_{\sigma y}\right) \\
& =m\left(\Phi \cap \sigma^{-1} \Omega \cap Y_{y}\right) \\
& >0
\end{aligned}
$$

which means that $\sigma y \in T$, because $\xi_{\sigma \Phi \cap \Omega} \in L_{\Omega}$ and $\xi_{\Phi^{-1} y \cap Y} \in L_{Y}$.

5.3. THEOREM. Let $\Omega \subseteq \Gamma$ and $Y \subseteq X$ be measurable relatively sigma-compact sets. Assume also that $L_{\Omega} * L_{Y} \subseteq L_{Y}$. Then there exist $\Omega_{1} \subseteq \Gamma$ and $Y_{1} \subseteq X$ such that $\Omega_{1}=\Omega$ a.e. and $Y_{1}=Y$ a.e., and such that $\Omega_{1} Y_{1} \subseteq Y_{1}$.

Proof. By the preceding theorem, $\Omega_{0} Y_{0}=T$. In view of the assumption $L_{\Omega} * L_{Y} \subseteq L_{Y}$ and the identity $L_{Y}=L_{Y_{0}}$, Theorem 4.14 tells us that $T \subseteq Y_{0}$ 1.a.e. Since $T$ is $\mathcal{O}$-open, $T \subseteq i_{\mathcal{O}} Y_{0}$. But then $\left(d \Omega_{0}\right) T \subseteq T$ by Lemma 4.13(ii). Now let $\Omega_{1}=\Omega_{0} \cap d \Omega_{0}$ and $Y_{1}=Y_{0} \cup T$. Then $\Omega_{1}=\Omega$ 1.a.e., $Y_{1}=Y$ 1.a.e., and $\Omega_{1} Y_{1} \subseteq \Omega_{0} Y_{0} \cup\left(d \Omega_{0}\right) T \subseteq T \subseteq Y_{1}$.

We mention that Theorem 5.3 generalizes Theorem 4.3 of [6]. We do not know if we may eliminate the sigma-compactness hypothesis. Under certain circumstances-when $Y$ is either open or closed-we can, as demonstrated in Corollaries 3.12 and 3.13 .

As might be expected, if we further restrict our attention to those $\Omega \subseteq \Gamma$ and $Y \subseteq X$ for which $L_{\Omega} * L_{Y}=L_{Y}$, we can furnish more explicit information. For this case we give a complete solution to the converse of Theorem 5.1. First we prove the theorem for $X=\Gamma$.

5.4. THEOREM. $L_{\Omega} * L_{\Omega}=L_{\Omega}$ if and only if there is an open set $\Omega_{0}$ in $\Gamma$ such that $\Omega_{0}=\Omega$ l.a.e. and such that $\Omega_{0} \Omega_{0}=\Omega_{0}$.

Proof. Assume that $L_{\Omega} * L_{\Omega}=L_{\Omega}$. We will show that we can take $\Omega_{0}$ to be $T$. By Theorem 4.14, $T=\Omega$ l.a.e., by Lemma 4.12(i) $T$ is open $(\mathcal{O}=\mathscr{T}$ !). In order that the definition of $\Omega_{0}$ as $T$ satisfy the conclusions of the theorem, we need only show that $T T=T$. Since

$$
T T \subseteq\left(i_{\mathcal{O}} T\right)\left(i_{\mathcal{O}} T\right)=\left(i_{\mathcal{O}} \Omega\right)\left(i_{\mathcal{O}} T\right) \subseteq\left(d_{\mathcal{O}} \Omega\right)\left(i_{\mathcal{O}} T\right),
$$

Lemma 4.13(ii) yields $T T \subseteq T$, whereas $T \subseteq T T$ by Lemma 4.12(ii) since $L_{T}=L_{\Omega}$.

To prove the converse, we first show that $\Omega=T$ 1.a.e. By Lemma 4.12(ii) $T \subseteq \Omega_{0} \Omega_{0}=\Omega_{0}$, while by Lemma 4.13(ii), $\Omega_{0} \Omega_{0} \subseteq T$, since $\Omega_{0}$ is open. Then Theorem 4.14 wraps it up: $L_{\Omega} * L_{\Omega}=L_{T}=L_{\Omega}$.

5.5. TheOREM. $L_{\Omega} * L_{Y}=L_{Y}$ if and only if there exist $\Omega_{0} \subseteq \Omega$ and 0 -open $Y_{0} \subseteq X$ such that $\Omega_{0}=\Omega$ l.a.e., $Y_{0}=Y$ l.a.e., and such that $\Omega_{0} Y_{0}=Y_{0}$. 
Proof. We show that $\Omega_{0}=\Omega \cap d \Omega$ and $Y_{0}=T$ satisfy the requirements, under the assumption that $L_{\Omega} * L_{Y}=L_{Y}$. Clearly $\Omega_{0}=\Omega$ 1.a.e. By virtue of Theorem 4.14, $T=Y$ 1.a.e. This means that $L_{\Omega_{0}} * L_{T}=L_{T}$, so that by Lemma 4.12(ii) $T \subseteq \Omega_{0} T$. On the other hand, $\Omega_{0} T \subseteq(d \Omega)\left(i_{\mathcal{O}} T\right)=(d \Omega)\left(i_{\mathcal{O}} Y\right) \subseteq T$ by Lemma 4.13(ii). The converse can be proved virtually the same as the converse in Theorem 5.4.

Assuredly, if $L_{\Gamma} \circ L_{X}^{\infty} \subseteq C(X)$, then $T$ is open in $\mathscr{T}$, so we obtain $Y_{0}$ open in that topology.

If $1 \in d \Omega$, then by Lemma 3.1, $L_{\Omega}$ contains an approximate identity $\left(u_{i}\right)_{i \in I}$ of $L_{\Gamma}$. For every $k \in L_{Y}$ we then have $k=\lim _{i} u_{i} * k \in L_{\Omega} * L_{Y}$. Thus we obtain

5.6. THEOREM. If $1 \in d \Omega$ and $L_{\Omega} * L_{Y} \subseteq L_{Y}$, then $L_{\Omega} * L_{Y}=L_{Y}$.

5.7. Corollary. Let $\Omega \subseteq \Gamma$ be such that $1 \in d \Omega$ and $L_{\Omega}$ is a subalgebra of $L_{\Gamma}$. Then there is an open $\Omega_{0} \subseteq T$ such that $\Omega_{0}=\Omega$ l.a.e. and $\Omega_{0} \Omega_{0}=\Omega_{0}$.

It is worth noticing that $\Gamma$ may well contain open subsets $\Omega$ with $\Omega \Omega=\Omega$ while $1 \notin d \Omega$. For an example, let $\Gamma$ be the additive group of the reals with the discrete topology, and $\Omega=\{\sigma \in \Gamma: \sigma>0\}$.

\section{REFERENCES}

1. N. Bourbaki, Intégration. Chapitre 6: Intégration vectorielle, Actualités Sci. Indust., no. 1281, Hermann, Paris, 1959. MR 23 \#A2033.

2. P. J. Cohen, Factorization in group algebras, Duke Math. J. 26 (1959), 199-205. MR 21 \#3729.

3. S. L. Gulick, T.-S. Liu and A. C. M. van Rooij, Group algebra modules. I, Canad. J. Math. 19 (1967), 133-150. MR 36 \#5712.

4. — Group algebra modules. II, Canad. J. Math. 19 (1967), 151-173. MR 36 \#5713.

5. E. Hewitt and K. A. Ross, Abstract harmonic analysis. Vol. 1: Structure of topological groups. Integration theory, group representations, Die Grundlehren der math. Wissenschaften, Band 115, Academic Press, New York and Springer-Verlag, Berlin and New York, 1963. MR 28 \#158.

6. T.-S. Liu, Invariant subspaces of some function spaces, Quart. J. Math. Oxford Ser. (2) 14 (1963), 231-239. MR 27 \#1561.

7. T.-S. Liu and A. C. M. van Rooij, Transformation groups and absolutely continuous measures. II, Nederl. Akad. Wetensch. Proc. Ser. A 73 =Indag. Math. 32 (1970), 57-61.

8. - Sums and intersections of normed linear spaces, Math. Nachr. 42 (1969), 29-42.

9. W. Rudin, Measure algebras on abelian groups, Bull. Amer. Math. Soc. 65 (1959), 227-247. MR 21 \#7404.

10. A. B. Simon, Vanishing algebras, Trans. Amer. Math. Soc. 92 (1959), 154-167. MR 23 \#A1240.

UNIVERSITY OF MARYLAND,

College Park, Maryland 20742

UNIVERSITY OF MASSACHUSETTS,

AMHERST, MASSACHUSETTS 01002

CATHOLIC UNIVERSity,

Nijmegen, The Netherlands 